\title{
The formation of the anorthosite lunar crust: a mineral dissolution model
}

\author{
DAN ZHU
}

State Key Laboratory of Ore Deposit Geochemistry, Institute of Geochemistry, Chinese Academy of Sciences, Guiyang 550081, China. Email: zhudan@vip.gyig.ac.cn

The origin of the anorthosite lunar crust is still elusive, although many models have been proposed. Here I present a model to quantitatively show that the lunar anorthosite was produced by kinetic (or disequilibrium) dissolution of gabbronorite, which crystallized on the surface of the lunar magma ocean (LMO) when the melt on the surface of the LMO was saturated in plagioclase with olivine and pyroxene during the temperature of the transient atmosphere was around $1300{ }^{\circ} \mathrm{C}$. The crystallized gabbronorite with significant grain size, before or during foundering into the LMO, was continually heated by the super-liquidus magma coming from the interior of the LMO due to the violent convection in the LMO. Because the dissolution rate of plagioclase is less than those of olivine and pyroxene according to the theory (Zhang et al., 1989) and the experimental results of mineral diffusive dissolution (Chen and Zhang, 2008; Chen and Zhang, 2009; Yu et al., 2016), and the dissolution process was short-lived, thus the melts produced by the dissolution were not fully in equilibrium with every kind of minerals. Therefore, the dissolution resulted in formation of the plagioclase-rich floating "rockbergs", since the non-cotectic melt produced by the kinetic dissolution, which is denser than plagioclase, would sank into the LMO. The thickening of the lunar crust was created by the kinetic dissolution of the gabbronorite crystallized at the base of the anorthosite lunar crust until the temperature at the base of the lunar crust was similar to that of the LMO's interior, thus a noritic composition of the lower lunar crust is a prediction, which is consistent with the noritic composition of the floor materials in the South Pole-Aitken basin (Pieters et al., 1997). The top-down growth of the lunar crust, fundamentally different from previous models, is consistent with the absence of plagioclase in the lunar mantle (O'Hara and Niu, 2015). Also, the kinetic model can explain not only many problems of the lunar crust, such as the age (ElkinsTanton, 2012), the asymmetry (Ohtake et al., 2012; Wieczorek et al., 2013), the chemical heterogeneity (PernetFisher et al., 2019) and the coexistence of ferroan and magnesian anorthosite (Gross et al., 2014), but also the formation of the anorthosite in the layered intrusion, which is also not very clear (Cawthorn and Ashwal, 2009). 